\title{
DESCRIPTION OF ZERO SEQUENCES FOR HOLOMORPHIC AND MEROMORPHIC FUNCTIONS OF FINITE $\lambda$-TYPE IN A CLOSED HALF-STRIP
}

\section{N.B. SOKULSKA}

\begin{abstract}
We describe the zero sets of holomorphic and meromorphic functions $f$ of finite $\lambda$-type in a closed half-strip satisfying $f(\sigma)=f(\sigma+2 \pi i)$ on the boundary.
\end{abstract}

Keywords: holomorphic function, meromorphic function, function of finite $\lambda$ - type, sequence of finite $\lambda$-density, $\lambda$-admissible sequence

Mathematics Subject Classification: 30D35

\section{INTRODUCTION}

Let $f$ be a meromorphic function in the closure of the half-strip

$$
S=\{s=\sigma+i t: \sigma>0, \quad 0<t<2 \pi\} .
$$

Suppose $f$ has neither zeros nor poles on $\partial S$, and $f(\sigma)=f(\sigma+2 \pi i), \sigma \geq 0$. Denote by $\left\{s_{j}\right\}$ the zero sequence of function $f$ in $S, s_{j}=\sigma_{j}+i t_{j}$, by $\left\{p_{j}\right\}$ the sequence of its poles in $S$.

Let $S^{*}$ be the strip $S$ with the straight slits $\left\{\tau \sigma_{j}+i t_{j}\right\},\left\{\tau \operatorname{Re} p_{j}+i \operatorname{Im} p_{j}\right\}, \quad 1 \leqslant \tau<\infty$. Given $s_{0} \in S^{*}$, suppose $\log f\left(s_{0}\right)$ is well-defined and let

$$
\log f(s)=\log f\left(s_{0}\right)+\int_{s_{0}}^{s} \frac{f^{\prime}(\zeta)}{f(\zeta)} d \zeta
$$

where integral is taken along a piecewise-smooth path in $S^{*} \cup \partial S$, which connects $s_{0}$ and $s$.

By $n(\eta, f)$ we denote the counting function of poles of $f$ in the rectangle $R_{\eta}=\{\sigma+i t: 0<$ $\sigma \leqslant \eta, \quad 0 \leqslant t<2 \pi\}$. We let

$$
N(\sigma, f)=\int_{0}^{\sigma} n(\eta, f) d \eta .
$$

and

$$
c_{0}(\sigma, f)=\frac{1}{2 \pi} \int_{0}^{2 \pi} \log |f(\sigma+i t)| d t .
$$

The following Lemma is a counterpart of Jensen-Littlewood Theorem ([1]).

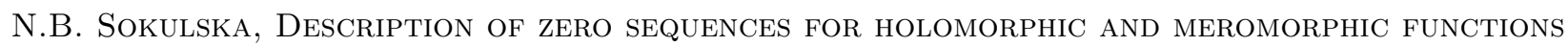
OF FINITE $\lambda$-TYPE IN A CLOSED HALF-STRIP.

(C) SOKUlska N.B. 2014.

Submitted January 24, 2014. 
Lemma 1. [2] Let $f$ be a meromorphic function in the closure of half-strip $S$, $f(\sigma)=f(\sigma+2 \pi i), \sigma \geq 0$. Then

$$
\begin{array}{r}
N\left(\sigma, \frac{1}{f}\right)-N(\sigma, f)=c_{0}(\sigma, f)-\frac{\sigma}{\sigma_{0}} c_{0}\left(\sigma_{0}, f\right)+\left(\frac{\sigma}{\sigma_{0}}-1\right) c_{0}(0, f), \\
\sigma \geq \sigma_{0}>0 .
\end{array}
$$

The Nevanlinna characteristic of such functions was defined in 2] as

$$
T(\sigma, f)=m_{0}(\sigma, f)-\frac{\sigma}{\sigma_{0}} m_{0}\left(\sigma_{0}, f\right)+\left(\frac{\sigma}{\sigma_{0}}-1\right) m_{0}(0, f)+N(\sigma, f), \quad \sigma \geq \sigma_{0}>0,
$$

where

$$
m_{0}(\sigma, f)=\frac{1}{2 \pi} \int_{0}^{2 \pi} \log ^{+}|f(\sigma+i t)| d t .
$$

Definition 1. A positive non-decreasing continuous unbounded function $\lambda(\sigma)$ defined for all $\sigma \geq \sigma_{0}>0$ is said to be a growth function.

Definition 2. Let $\lambda(\sigma)$ be a growth function and $f$ be a meromorphic function in $\bar{S}$, such that $f(\sigma+2 \pi i)=f(\sigma), \sigma \geq \sigma_{0}>0$. We say that $f$ is of finite $\lambda$-type if $T(\sigma, f) \leqslant A \lambda(\sigma+B)$, $\sigma \geq \sigma_{0}$ for some constants $A>0, B>0$ and all $\sigma, \sigma \geq \sigma_{0}>0$.

We denote by $\Lambda$ the class of meromorphic functions of finite $\lambda$-type in $\bar{S}$ and $\Lambda_{H}$ the class of holomorphic functions of finite $\lambda$-type in $\bar{S}$.

In this paper we describe the zero sequences of holomorphic functions in $\Lambda_{H}$, as well as zero and pole sequences of meromorphic functions in $\Lambda$.

For entire and meromorphic in $\mathbb{C}$ functions similar problems were solved by L. Rubel and B. Taylor ([3]), for holomorphic and meromorphic functions in a punctured plane the same was done by A. Kondratyuk and I. Laine ([4]).

\section{DESCRIPTION OF ZERO SEQUENCES OF HOLOMORPHIC AND MEROMORPHIC FUNCTIONS OF FINITE $\lambda$-TYPE IN A HALF-STRIP}

Let $Q=\left\{s_{j}\right\}$ be a sequence of complex numbers in $\bar{S}$. By $n(\eta, Q)$ we indicate the counting function of $Q$ in the rectangle $R_{\eta}$ and we let

$$
N(\sigma, Q)=\int_{0}^{\sigma} n(\eta, Q) d \eta
$$

Definition 3. A sequence $Q=\left\{s_{j}\right\}$ from $\bar{S}$ has a finite $\lambda$-density if

$$
N(\sigma, Q) \leqslant A \lambda(\sigma+B)
$$

for some positive constants $A, B$ and all $\sigma, \sigma \geq \sigma_{0}>0$.

Definition 4. A sequence $Q=\left\{s_{j}\right\}$ from $\bar{S}$ is said to be $\lambda$-admissible if it has finite $\lambda$-density and there are positive constants $A, B$ such that

$$
\frac{1}{k}\left|\sum_{\sigma_{1}<\operatorname{Re}_{s_{j} \leqslant \sigma_{2}}}\left(\frac{1}{e^{s_{j}}}\right)^{k}\right| \leqslant \frac{A \lambda\left(\sigma_{1}+B\right)}{e^{k \sigma_{1}}}+\frac{A \lambda\left(\sigma_{2}+B\right)}{e^{k \sigma_{2}}},
$$

for all $\sigma_{1}, \sigma_{2}, \sigma_{0} \leqslant \sigma_{1}<\sigma_{2}$ and each $k \in \mathbb{N}$. 
Denote

$$
c_{k}(\sigma, f)=\frac{1}{2 \pi} \int_{0}^{2 \pi} e^{-i k t} \log |f(\sigma+i t)| d t, \quad k \in \mathbb{Z} .
$$

For a meromorphic in $\bar{R}_{\sigma}$ function $f$ such that $f(\sigma)=f(\sigma+2 \pi i)$ the following relations hold true (see [2]):

$$
\begin{aligned}
c_{k}(\sigma, f)= & \frac{e^{k \sigma}}{2 k} \alpha_{k}(f)-\frac{e^{-k \sigma}}{2 k} \overline{\alpha_{-k}}(f) \\
& +\frac{1}{2 k} \sum_{s_{j} \in R_{\sigma}}\left[\left(\frac{e^{\sigma}}{e^{s_{j}}}\right)^{k}-\left(\frac{e^{\overline{s_{j}}}}{e^{\sigma}}\right)^{k}\right]-\frac{1}{2 k} \sum_{p_{j} \in R_{\sigma}}\left[\left(\frac{e^{\sigma}}{e^{p_{j}}}\right)^{k}-\left(\frac{e^{\overline{p_{j}}}}{e^{\sigma}}\right)^{k}\right], \\
c_{-k}(\sigma, f)= & \bar{c}_{k}(\sigma, f) \quad k \in \mathbb{N},
\end{aligned}
$$

where $s_{j}, p_{j}$ are its zeroes and poles in $\bar{R}_{\sigma}$ respectively, and

$$
\alpha(f)=\frac{1}{2 \pi} \int_{0}^{2 \pi} e^{-i k t} \frac{f^{\prime}(i t)}{f(i t)} d t, \quad k \in \mathbb{N} .
$$

Theorem 1. A sequence $Q$ in $\bar{S}$ is the zero sequence of the function in $\Lambda_{H}$ if and only if it is $\lambda$-admissible.

Proof. Let $Q=\left\{s_{j}\right\}$ be the zero sequence of a function $f$ from $\Lambda_{H}$. Then by (6)

$$
\begin{aligned}
\frac{c_{k}\left(\sigma_{2}, f\right)}{e^{k \sigma_{2}}}-\frac{c_{k}\left(\sigma_{1}, f\right)}{e^{k \sigma_{1}}}= & \frac{\alpha_{k} e^{k \sigma_{2}}-\overline{\alpha_{-k}} e^{-k \sigma_{2}}}{2 k e^{k \sigma_{2}}}+\frac{1}{2 k e^{k \sigma_{2}}}\left[\sum_{s_{j} \in R_{\sigma_{2}}}\left(\frac{e^{\sigma_{2}}}{e^{s_{j}}}\right)^{k}-\sum_{s_{j} \in R_{\sigma_{2}}}\left(\frac{e^{\overline{s_{j}}}}{e^{\sigma_{2}}}\right)^{k}\right] \\
& -\frac{\alpha_{k} e^{k \sigma_{1}}-\overline{\alpha_{-k}} e^{-k \sigma_{1}}}{2 k e^{k \sigma_{1}}}-\frac{1}{2 k e^{k \sigma_{1}}}\left[\sum_{s_{j} \in R_{\sigma_{1}}}\left(\frac{e^{\sigma_{1}}}{e^{s_{j}}}\right)^{k}-\sum_{s_{j} \in R_{\sigma_{1}}}\left(\frac{e^{\overline{s_{j}}}}{e^{\sigma_{1}}}\right)^{k}\right] \\
= & \frac{\overline{\alpha_{-k}}}{2 k}\left[\frac{1}{e^{2 k \sigma_{1}}}-\frac{1}{e^{2 k \sigma_{2}}}\right]+\frac{1}{2 k}\left[\sum_{s_{j} \in R_{\sigma_{2}}} \frac{1}{\left(e^{s_{j}}\right)^{k}}-\sum_{s_{j} \in R_{\sigma_{1}}} \frac{1}{\left(e^{s_{j}}\right)^{k}}\right] \\
& +\frac{1}{2 k e^{k \sigma_{1}}} \sum_{s_{j} \in R_{\sigma_{1}}}\left(\frac{e^{\overline{s_{j}}}}{e^{\sigma_{1}}}\right)^{k}-\frac{1}{2 k e^{k \sigma_{2}}} \sum_{s_{j} \in R_{\sigma_{2}}}\left(\frac{e^{\overline{s_{j}}}}{e^{\sigma_{2}}}\right)^{k},
\end{aligned}
$$

where $0 \leqslant \sigma_{1}<\sigma_{2}$.

Then we obtain

$$
\begin{aligned}
\frac{1}{k} \sum_{\sigma_{1}<\operatorname{Re}_{s_{j} \leqslant \sigma_{2}}} \frac{1}{\left(e^{s_{j}}\right)^{k}}= & \frac{2 c_{k}\left(\sigma_{2}, f\right)}{e^{k \sigma_{2}}}-\frac{2 c_{k}\left(\sigma_{1}, f\right)}{e^{k \sigma_{1}}}+\frac{\overline{\alpha_{-k}}}{k}\left[\frac{1}{e^{2 k \sigma_{2}}}-\frac{1}{e^{2 k \sigma_{1}}}\right]+ \\
& +\frac{1}{k e^{k \sigma_{2}}} \sum_{s_{j} \in R_{\sigma_{2}}}\left(\frac{e^{\overline{s_{j}}}}{e^{\sigma_{2}}}\right)^{k}-\frac{1}{k e^{k \sigma_{1}}} \sum_{s_{j} \in R_{\sigma_{1}}}\left(\frac{e^{\overline{s_{j}}}}{e^{\sigma_{1}}}\right)^{k}
\end{aligned}
$$

We have

$$
\sum_{s_{j} \in R_{\sigma_{i}}}\left|\frac{e^{\overline{s_{j}}}}{e^{\sigma_{i}}}\right|^{k} \leqslant \sum_{s_{j} \in R_{\sigma_{i}}} 1 \leqslant n\left(\sigma_{i}+1, \frac{1}{f}\right) \leqslant N\left(\sigma_{i}+1, \frac{1}{f}\right) \leqslant A_{1} \lambda\left(\sigma_{i}+1+B_{1}\right), \quad \sigma_{i} \in R_{\sigma_{i}}, i=1,2,
$$

for some constants $A_{1}, B_{1}>0$. 
We also get the estimate for the left-hand side of identity (7):

$$
\begin{aligned}
& \frac{1}{k} \sum_{\sigma_{1}<\operatorname{Re}_{s_{j} \leqslant \sigma_{2}} \frac{1}{e^{k s_{j}}} \mid \leqslant} \frac{A_{2} \lambda\left(\sigma_{2}+B_{2}\right)}{e^{k \sigma_{2}}}+\frac{A_{2} \lambda\left(\sigma_{1}+B_{2}\right)}{e^{k \sigma_{1}}}+\frac{\left|\overline{\alpha_{-k}}\right|}{k}\left\lceil\frac{1}{e^{2 k \sigma_{2}}}+\frac{1}{e^{2 k \sigma_{1}}}\right] \\
&+\frac{1}{k e^{k \sigma_{2}}} \sum_{s_{j} \in R_{\sigma_{2}}}\left|\frac{e^{\overline{s_{j}}}}{e^{\sigma_{2}}}\right|^{k}+\frac{1}{k e^{k \sigma_{1}}} \sum_{s_{j} \in R_{\sigma_{1}}}\left|\frac{e^{\overline{s_{j}}}}{e^{\sigma_{1}}}\right|^{k} \\
& \leqslant \frac{A_{2} \lambda\left(\sigma_{2}+B_{2}\right)}{e^{k \sigma_{2}}}+\frac{A_{2} \lambda\left(\sigma_{1}+B_{2}\right)}{e^{k \sigma_{1}}}+C\left[\frac{1}{e^{2 k \sigma_{2}}}+\frac{1}{e^{2 k \sigma_{1}}}\right] \\
&+\frac{1}{k e^{k \sigma_{2}}} N\left(\sigma_{2}+1, \frac{1}{f}\right)+\frac{1}{k e^{k \sigma_{1}}} N\left(\sigma_{1}+1, \frac{1}{f}\right) \\
& \leqslant \frac{A \lambda\left(\sigma_{2}+B\right)}{e^{k \sigma_{2}}}+\frac{A \lambda\left(\sigma_{1}+B\right)}{e^{k \sigma_{1}}}, \quad k \in \mathbb{N}, \quad \sigma_{2}>\sigma_{1} \geqslant \sigma_{0},
\end{aligned}
$$

where $A=\max \left\{A_{1}, A_{2}, C\right\}, B=\max \left\{B_{1}+1, B_{2}\right\}$.

Theorem 2 in $[2$ implies that the sequence $Q$ has a finite $\lambda$-density. Hence, it is $\lambda$-admissible.

Let now $Q=\left\{s_{j}\right\}$ be $\lambda$-admissible. Then the sequence $Z=\left\{z_{j}\right\}, z_{j}=e^{s_{j}} \in \mathbb{C}$, is $\lambda_{1^{-}}$ admissible in $\mathbb{C}$, where $\lambda_{1}(r)=\lambda(\log r)$. By the Rubel-Taylor Theorem [3, p. 84], (see also [5. p. 29]), there exists an entire function $F(z)$ of finite $\lambda_{1}$-type with zero sequence $Z=\left\{z_{j}\right\}$. Therefore, the function $f(s)=F\left(e^{s}\right)$ is holomorphic of finite $\lambda$-type in $\bar{S}$ with the zero sequence $\left\{s_{j}\right\}$.

Theorem 2. A sequence $Q$ in $\bar{S}$ is the zero sequence of a function in $\Lambda$ if and only if it has finite $\lambda$-density.

Proof. If $Q=\left\{s_{j}\right\}$ is the zero sequence of a function $f, f \in \Lambda$, then from [2], we have

$$
N(\sigma, Q)=N\left(\sigma, \frac{1}{f}\right) \leqslant T(\sigma, f) \leqslant B \lambda(\sigma+C)
$$

for all $\sigma \geqslant \sigma_{0}>0$ and some $B, C>0$.

Let now $Q=\left\{s_{j}\right\}$ be a sequence of finite $\lambda$-density. Then the sequence $Z=\left\{z_{j}\right\}, \quad z_{j}=e^{s_{j}}$, has the finite $\lambda_{1}$-density if $\lambda_{1}(r)=\lambda(\log r)$. By the Rubel-Taylor Theorem [3, p. 88] (see also [5, p. 35]) there exist a meromorphic function $F$ of finite $\lambda_{1}$-type with zero sequence $Z$. The function $f(s)=F\left(e^{s}\right)$ is the meromorphic of finite $\lambda$-type in $\bar{S}$ with zero sequence $\left\{s_{j}\right\}$.

Corollary 1. A sequence $P=\left\{p_{j}\right\}$ is the pole sequence of a function $f$ from $\Lambda$ if and only if it has finite $\lambda$-density.

Proof. Apply Theorem 2 to the function $\frac{1}{f}$. 


\section{BIBLIOGRAPHY}

1. J.E. Littlewood On the zeros of the Riemann zeta-function, Proc. Camb. Philos. Soc. 22 (1924), 295-318.

2. N.B. Sokul's'ka Meromorphic functions of finite $\lambda$-type in half-strip // Carpathian Mathematical Publications 2012. V.4, 2. P. 328-339 (in Ukrainian).

3. L.A. Rubel, B.A. Taylor Fourier series method for meromorphic functions // Bull. Soc. Math. France 1968. 96. P. 53-96.

4. A. Kondratyuk, I. Laine Meromorphic functions in multiply connected domains // Fourier series method in complex analysis (Merkrijärvi, 2005),Univ. Joensuu Dept. Math. Rep. Ser., 10 (2006), P. 9-111.

5. A.A. Kondratyuk Fourier Series and Meromorphic Functions. L'viv.: Izdat. L'viv Univ., 1988. 196 p (in Russian).

Natalia Bogdanovna Sokulska,

Ivan Franko National University of L'viv,

Universytets'ka str. 1,

79000, Lviv, Ukraine

E-mail: natalya.sokulska@gmail.com 